STUDI

FRANCESI

\section{Studi Francesi}

Rivista quadrimestrale fondata da Franco Simone

165 (LV | III) | 2011

LA RAPPRESENTAZIONE DELLA MADRE NELLA

LETTERATURA FRANCESE DEL NOVECENTO a cura di Dario Cecchetti e Michele Mastroianni

\title{
Les Écrivains suisses et «La Nouvelle Revue française», études réunies par Daniel Maggetti
}

\section{Gian Luigi Di Bernardini}

\section{(2) OpenEdition \\ Journals}

Edizione digitale

URL: http://journals.openedition.org/studifrancesi/5150

DOI: 10.4000/studifrancesi.5150

ISSN: 2421-5856

Editore

Rosenberg \& Sellier

Edizione cartacea

Data di pubblicazione: 1 décembre 2011

Paginazione: 679-680

ISSN: 0039-2944

Notizia bibliografica digitale

Gian Luigi Di Bernardini, «Les Écrivains suisses et «La Nouvelle Revue française», études réunies par

Daniel Maggetti», Studi Francesi [Online], 165 (LV | III) | 2011, online dal 30 novembre 2015, consultato il

13 janvier 2021. URL: http://journals.openedition.org/studifrancesi/5150 ; DOI: https://doi.org/

10.4000/studifrancesi.5150

Questo documento è stato generato automaticamente il 13 janvier 2021.

\section{(†) $\ominus$

Studi Francesi è distribuita con Licenza Creative Commons Attribuzione - Non commerciale - Non opere derivate 4.0 Internazionale. 


\title{
Les Écrivains suisses et «La Nouvelle Revue française», études réunies par Daniel Maggetti
}

\author{
Gian Luigi Di Bernardini
}

\section{NOTIZIA}

Les Écrivains suisses et «La Nouvelle Revue française», études réunies par Daniel MAGGETTI, Paris, Classiques Garnier, 2009, pp. 252.

1 Daniel Maggetti, curatore del volume, spiega chiaramente nella sua introduzione (Des voisins peu causants: les écrivains suisses dans la NRF, pp. 9-17) come lo studio dei rapporti intercorsi tra la «Nouvelle Revue Française», il cui ruolo è ben noto e non dev'essere qui ricordato, e gli scrittori svizzeri francofoni finisca per fornire molte indicazioni sulle linee guida tenute dai redattori della rivista fondata da Gide nel 1909, più che sui percorsi dei singoli scrittori presi in esame.

2 Studio necessariamente parziale per ragioni intrinseche (figure come quella di Cendrars, di Robert Pinget o di Albert Cohen sono infatti state escluse per via della loro appartenenza alla rete produttivo-culturale francese), questo testo offre un'ulteriore occasione per verificare l'esistenza del principale punto identitario della «Nouvelle Revue Française», ovvero la sua coerente battaglia per una rigorosa estetica classicista, senza tuttavia dimenticare un'intelligente e costante attenzione alle avanguardie.

Il tema della collaborazione con scrittori come Ramuz, Cingria, Rougemont, Chessex mette in luce, però, anche un aspetto meno evidente dell'identità della rivista $\mathrm{e}$, in particolar modo, quello di una visione della letteratura europea di lingua francese esclusivamente imperniata su Parigi, in cui le istituzioni di questa città (riviste, premi letterari, ecc.) appaiono le uniche dotate di sufficiente autorità per legittimare la validità di questo o quell'autore francofono. Il tema affrontato in questo volume ripropone, insomma, il classico dissidio tra centro e periferia caratteristico dei rapporti 
tra le letterature e culture di lingua francese in ambito europeo e, a ben guardare, mondiale.

4 Numerosi sono i contributi monografici che costituiscono l'ossatura del testo e si soffermano, oltre a quelle già citate, anche su figure come quella di Chessex o dei critici dell'école de Genève (Poulet, Rousset, Starobinski). Vorremmo tuttavia citare in modo particolare l'ultimo articolo di Valérie cossY, Femmes écrivains: la cohorte absente (pp. 213-242), che si occupa in modo specifico del contributo dato da alcune scrittrici svizzero-francofone (Alice Rivaz, Catherine Colomb e Corinna Bille in particolare) il cui riconoscimento è stato doppiamente difficile, sia in quanto donne, sia a causa della loro origine svizzera. 